\title{
THREE-DIMENSIONAL NUMERICAL MODEL FOR SIMULATING THE ACCUMULATION PROCESS OF IMMERSED TUBE TANK OF HMZ BRIDGE
}

\author{
Wang Zhili ${ }^{1}$, Geng Yanfen ${ }^{2}$ and Lu Yongjun ${ }^{3}$
}

\begin{abstract}
In this paper, the development and implementation of a three-dimensional, numerical sediment transport model, which is based on staggered C-unstructured grids in the horizontal direction and Z-level grids in the vertical direction, is delineated. The three dimensional model is discretized by semi-implicit finite volume method, in that the free-surface and vertical diffusion are semi-implicit, thereby removing stability limitations associated with the surface gravity wave and vertical diffusion terms. The remaining terms in the momentum equations are discretized explicitly by integral method. The model is closed physically and mathematically using the Mellor and Yamada level-2.5 turbulent closure submodel. The numerical model is used for simulation accumulation process of immersed tube tank of HMZ (Hong Kong-Macau-Zhuhai) bridge. The model is calibrated and its performance extensively assessed against on-site experiment.
\end{abstract}

Keywords: 3D model; finite volume; unstructured grid; HMZ Bridge; tube tank; sediment transport

\section{INTRODUCTION}

The in-construction HMZ (Hong Kong-Zhuhai-Macau) Bridge is a series of bridges and tunnels that will connect the west side of Hong Kong to Macau and the mainland Chinese city of Zhuhai, which are situated on the west side of the Pearl River Delta and across the Lingding bay, in which immersed tube method will be used for the tunnel (see Fig.1). The immersed tube tank is about 5.76km long, $47 \mathrm{~m}$ wide, $10 \mathrm{~m}$ to $30 \mathrm{~m}$ deep. The accumulation process of the immersed tube tank is very important for tube tank project of HMZ bridge. For predicting the accumulation process, the on-site experiment is carried out near the west artificial island (see Fig.1), from February 2009 to July 2009, which is $100 \mathrm{~m}$ long, $21 \mathrm{~m}$ wide, about $12 \mathrm{~m}$ deep, 1:10 to 1:5 side slope (see Fig.2). The purpose of this study is to develop a three dimensional (3D) sediment transport model that could simulate the accumulation process of immersed tube tank of HMZ bridge.

Ocean and estuary sediment transport have been well predicted by using two dimensional depth average models in the horizontal plane (Lu et al, 2009; Ding and Wang, 2008). However, the area near the main structure of a project has 3D problems of water flow and sediment motion, which are too complex for 2D mathematical models. Therefore, 3D numerical model for sediment transport is needed. Three numerical methods have widely used in three dimensional ocean sediment transport model: (1) the finite difference method (Blumberg and Mellor, 1980, 1987; Zhang et al, 2004); finite element method (Lynch and Werner, 1991; Zhang and Baptista, 2008); and finite volume method (Chen el al, 2003. Fringer et al, 2006).

In this study, the three dimensional sediment transport model is developed by finite volume method on unstructured to be applicable to simulate he accumulation process of immersed tube tank of HMZ bridge. The model is investigated by comparing the compute results with on-site experiment data, and than used to predict the accumulation process of the immersed tube tank of HMZ bridge..

\footnotetext{
${ }^{1}$ State Key Laboratory of Hydrology-Water Resources and Hydraulic Engineering and Key Laboratory of Port, Waterway and Sedimentation Engineering of Ministry of Transport, Nanjing Hydraulic Research Institute, 34\# Hujuguan road,Nanjing,210024, China

${ }^{2}$ Transportation College, Southeast University,4\# Shipailou Road, Nanjing,210096, China

${ }^{3}$ State Key Laboratory of Hydrology-Water Resources and Hydraulic Engineering, Nanjing Hydraulic Research Institute, 34\# Hujuguan road,Nanjing,210024 ,China
} 


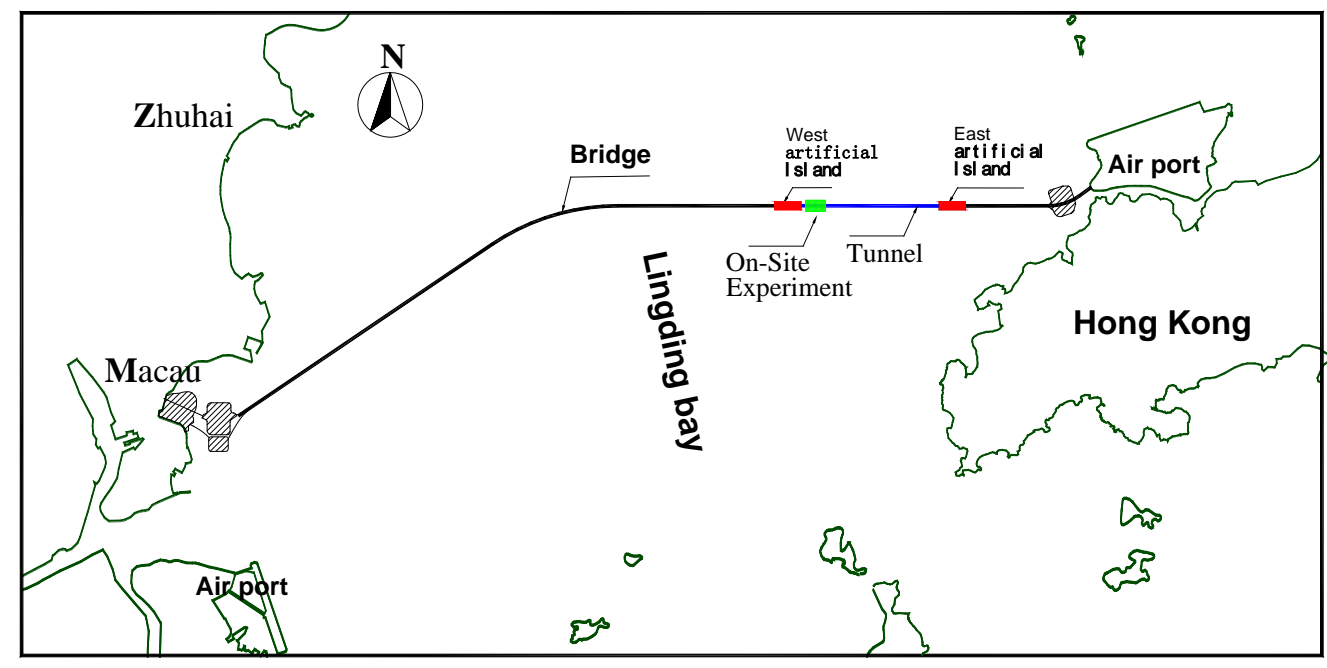

Fig.1 The sketch of Hong Kong-Macau-Zhuhai bridge

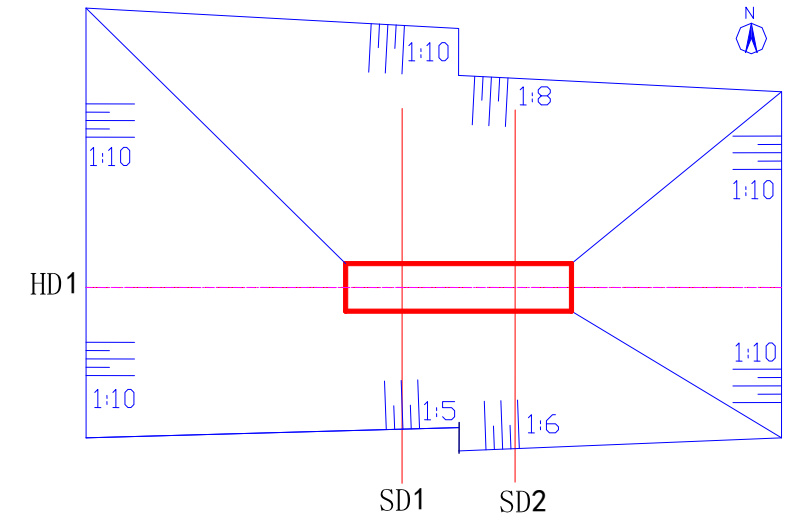

Fig.2 The sketch of scoop tank of on-Site Experiment

\section{NUMERICAL MODEL}

\subsection{Governing equations}

The governing equations consist of the following continuity, momentum, suspended sediment transport, density and bed deformation equations

$$
\begin{gathered}
\frac{\partial u}{\partial x}+\frac{\partial v}{\partial y}+\frac{\partial w}{\partial z}=0 \\
\frac{\partial u}{\partial t}+\frac{\partial u u}{\partial x}+\frac{\partial v u}{\partial y}+\frac{\partial w u}{\partial y}=f v-g \frac{\partial \eta}{\partial x}-\frac{g}{\rho_{0}} \int_{z_{b}}^{\eta} \frac{\partial \rho}{\partial x} d z \\
+\frac{\partial}{\partial x}\left(\gamma_{h} \frac{\partial u}{\partial x}\right)+\frac{\partial}{\partial y}\left(\gamma_{h} \frac{\partial u}{\partial y}\right)+\frac{\partial}{\partial z}\left(\gamma_{v} \frac{\partial u}{\partial z}\right) \\
\frac{\partial v}{\partial t}+\frac{\partial u v}{\partial x}+\frac{\partial v v}{\partial y}+\frac{\partial w v}{\partial y}=-f u-g \frac{\partial \eta}{\partial y}-\frac{g}{\rho_{0}} \int_{z_{b}}^{\eta} \frac{\partial \rho}{\partial y} d z \\
+\frac{\partial}{\partial x}\left(\gamma_{h} \frac{\partial v}{\partial x}\right)+\frac{\partial}{\partial y}\left(\gamma_{h} \frac{\partial v}{\partial y}\right)+\frac{\partial}{\partial z}\left(\gamma_{v} \frac{\partial v}{\partial z}\right)
\end{gathered}
$$




$$
\begin{gathered}
\frac{\partial s}{\partial t}+\frac{\partial u s}{\partial x}+\frac{\partial v s}{\partial y}+\frac{\partial w s}{\partial y}= \\
+\frac{\partial}{\partial x}\left(K_{h} \frac{\partial s}{\partial x}\right)+\frac{\partial}{\partial y}\left(K_{h} \frac{\partial s}{\partial y}\right)+\frac{\partial}{\partial z}\left(K_{v} \frac{\partial s}{\partial z}\right) \\
\rho=\rho(s) \\
\gamma_{s} \frac{\partial z_{b}}{\partial t}=\varpi\left(s_{b l}-s_{b l}^{*}\right)
\end{gathered}
$$

where $X, y$, and $z$ are the east, north, and vertical axes of the Cartesian coordinate; $u, v$, and $w$ are the $x, y, z$ velocity components; $\eta$ is the surface water level; $\rho$ is the density; $f$ is the Coriolis parameter; $g$ is the gravitational acceleration; $s$ is the sediment concentration; $s_{b l}$ and $s_{b l}^{*}$ are sediment concentration and sediment carrying capacity near the bed surface, respectively; $\omega$ is particle settling velocity of sediment; $z_{b}$ is the bed level; $\gamma_{s}$ is the dry unit weight of deposited sediment; $\gamma_{h}$ and $\gamma_{v}$ are the horizontal and vertical eddy viscosity coefficient, respectively; $K_{h}$ and $K_{v}$ are the sediment horizontal and vertical eddy diffusion coefficient, respectively.

Here, $\gamma_{h}$ and $K_{h}$ are parameterized using mixing length model. $\gamma_{v}$ and $K_{v}$ are parameterized using the Mellor and Yamada(1982) turbulent closure scheme. The equations for $k$ and $k l$ can be simplified as

$$
\begin{aligned}
& \frac{\partial k^{2}}{\partial t}+\frac{\partial u k^{2}}{\partial x}+\frac{\partial v k^{2}}{\partial y}+\frac{\partial w k^{2}}{\partial z}= \\
& \frac{\partial}{\partial x}\left(M_{h} \frac{\partial k^{2}}{\partial x}\right)+\frac{\partial}{\partial y}\left(M_{h} \frac{\partial k^{2}}{\partial y}\right)+\frac{\partial}{\partial z}\left(K_{v} \frac{\partial k^{2}}{\partial z}\right)+2\left(P_{s}+P_{b}-\varepsilon\right) \\
& \frac{\partial k^{2} l}{\partial t}+\frac{\partial u k^{2} l}{\partial x}+\frac{\partial v k^{2} l}{\partial y}+\frac{\partial w k^{2} l}{\partial z}= \\
& \frac{\partial}{\partial x}\left(M_{h} \frac{\partial k^{2} l}{\partial x}\right)+\frac{\partial}{\partial y}\left(M_{h} \frac{\partial k^{2} l}{\partial y}\right)+\frac{\partial}{\partial z}\left(K_{v} \frac{\partial k^{2} l}{\partial z}\right)+l E_{l}\left(P_{s}+P_{b}-\frac{W}{E_{l}} \varepsilon\right)
\end{aligned}
$$

where $k^{2}=0.5\left(u^{\prime 2}+v^{\prime 2}\right)$ is the turbulent kinetic energy; $l$ is the turbulent macroscale; $M_{h}$ and $M_{v}$ are the horizontal and vertical eddy diffusion coefficient of the turbulent kinetic energy; $P_{s}=K_{m}\left(u_{z}^{2}+v_{z}^{2}\right)$ and $P_{b}=\left(g K_{v} \rho_{z}\right) / \rho_{0}$ are the shear and buoyancy production terms of turbulent energy dissipation rate; $\varepsilon=k^{3} / B_{1} l$ is the turbulent kinetic energy dissipation rate; $W=1+E_{2} l^{2} /(\kappa L)^{2}$ is a wall proximity function, where $L^{-1}=(\xi-z)^{-1}+\left(z_{b}-z\right)^{-1}$; $\kappa=0.4$ is the von Karman constant.

The turbulent kinetic energy and macroscale equations are closed by defining

$$
\gamma_{v}=l k S_{m} ; K_{v}=l k S_{h} ; M_{k}=0.2 l k
$$

The stability functions $S_{M}$ and $S_{h}$ are defined as (Chen and Liu, 2003) 


$$
\begin{gathered}
S_{m}=\frac{0.4275-3.354 G_{h}}{\left(1-34.676 G_{h}\right)\left(1-6.127 G_{h}\right)} \\
S_{h}=\frac{0.494}{1-34.676 G_{h}}
\end{gathered}
$$

where $G_{h}=\left(l^{2} g / k^{2} \rho_{0}\right) \rho_{z} \cdot G_{h}$ has an upper bound of 0.023 for the case of unstable $\left(\rho_{z}>0\right)$ stratification and lower bound of -0.28 for the case of stable $\left(\rho_{z}<0\right)$ stratification.

\subsection{Boundary conditions}

The surface and bottom boundary conditions for $u v$, and $w$ are

$$
\begin{array}{r}
\gamma_{v}\left(\frac{\partial u}{\partial z}, \frac{\partial v}{\partial z}\right)=\frac{1}{\rho_{0}}\left(\tau_{s x}, \tau_{s y}\right) \text { at } z=\eta \\
w=\frac{\partial \zeta}{\partial t}+u \frac{\partial \zeta}{\partial x}+v \frac{\partial \zeta}{\partial y} \text { at } z=\eta \\
\gamma_{v}\left(\frac{\partial u}{\partial z}, \frac{\partial v}{\partial z}\right)=\frac{1}{\rho_{0}}\left(\tau_{b x}, \tau_{b y}\right) \text { at } z=z_{b} \\
w=u \frac{\partial z_{b}}{\partial x}+v \frac{\partial z_{b}}{\partial y} \quad \text { at } z=z_{b}
\end{array}
$$

Where $\left(\tau_{s x}, \tau_{s y}\right)$ and $\left(\tau_{b x}, \tau_{b y}\right)=C_{d} \sqrt{u^{2}+v^{2}}(u, v)$ are the $x$ and $y$ components of surface wind and bottom stresses. The drag coefficient $C_{d}$ is determined by matching a logarithmic bottom layer to the model at a height $\Delta z_{a b}$ above the bottom; that is

$$
C_{d}=\max \left(\frac{\kappa^{2}}{\ln \left(\Delta z_{a b} / z_{a}\right)^{2}}, 0.0025\right)
$$

Where $Z_{a}$ is the bottom roughness parameter.

There surface and bottom boundary condition for the turbulent kinetic energy and macroscale equations are

$$
\begin{aligned}
& k^{2} l=0, k^{2}=B_{1}^{2 / 3} u_{\tau s}^{2} \text { at } z=\eta \\
& k^{2} l=0, k^{2}=B_{1}^{2 / 3} u_{t b}^{2} \text { at } z=z_{b}
\end{aligned}
$$

Where $u_{\imath s}$ are the friction velocity associated with the surface and bottom stresses.

The surface and bottom boundary condition for the sediment transport are

$$
\begin{aligned}
& \omega s+K_{v} \frac{\partial s}{\partial z}=0 \quad \text { at } z=\eta \\
& \omega s+K_{v} \frac{\partial s}{\partial z}=D_{b}-E_{b} \text { at } z=z_{b}
\end{aligned}
$$

where $E_{b}$ and $D_{b}$ are deposition rate and erosion (resuspension) flux rate at bottom, respectively.

The depositional flux rate is computed using the following settling formulation for cohesive sediment, 


$$
D_{b}=\left\{\begin{array}{cl}
\omega s F\left(1-\tau_{b} / \tau_{c r, d}\right) & \tau_{b} \leq \tau_{c r, d} \\
0 & \tau_{b}>\tau_{c r, d}
\end{array}\right.
$$

where $\tau_{c r, d}$ is a critical bed shear stress and is between 0.06 and $0.10 \mathrm{~N} / \mathrm{m}^{2}$.

The erosion flux rate expressed as dry mass of mud eroded per unit area per unit time as a function of shear stress

$$
E_{b}=\left\{\begin{array}{cl}
M\left(\tau_{b} / \tau_{c r, e}-1\right) & \tau_{b}>\tau_{c r, e} \\
0 & \tau_{b} \leq \tau_{c r, e}
\end{array}\right.
$$

where $M$ is erodibility coefficient relating to the sediment properties, the values are in the range of $0.00001-0.00004 \mathrm{~kg} / \mathrm{m}^{2} s . \tau_{c r, e}$ is the critical bed shear stress for erosion.

\subsection{Unstructured grid}

We confine ourselves to three-dimensional z-level grids, for which vertical grid spacings remain constant in the horizontal. In plan, the domain is subdivided into a set of nonoverlapping unstructured cells. In plan, one has $N_{e}$ cells, each cell with index $i$, and each cell having an arbitrary number of faces $E_{i} \geq 3$. Let $N_{s}$ be the total number of faces. Each face with index $j$ has a predefined normal (whose orientation is arbitrary), $\boldsymbol{n}_{j}=\left(n_{x}, n_{y}\right)_{j}$, which indicates the positive direction of the velocity vector defined on that face, so that, if $\boldsymbol{u}_{j}=(u, v)_{j}$ is the velocity vector at face $j$, then the normal velocity to $j$ face is defined as

$$
U_{j}=\boldsymbol{u}_{j} \cdot \boldsymbol{n}_{j}
$$

The faces of the $i$ th cell are identified by an index $j(i, l), l=1,2, \cdots, E_{i}$. Similarly, the two cells that share the $j$ th face of the grid are identified by the indices $j 1$ and $j 2$, if $j$ face is the bourdary, we let $j 2=-1$. Without loss of generality, we can let normal of face $j$ have the same direction with $C_{j 1} C_{j 2}$. The outward-pointing normals of cell $i$ can be defined by $\boldsymbol{n}_{j(i, l)}^{\boldsymbol{o}}$ 。 Rather than storing the components of each of these normals, we store the dot product of the outward normal with the unique normal at every face $\boldsymbol{n}_{j}$, and define this as

$$
\mathrm{N}_{\mathrm{j}(\mathrm{i}, \mathrm{l})}=\boldsymbol{n}_{\boldsymbol{j}(\mathrm{i}, \mathrm{l})}^{\boldsymbol{o}} \cdot \boldsymbol{n}_{\boldsymbol{j}(\mathrm{i}, \boldsymbol{l})}= \pm 1
$$

From which we can get

$$
\boldsymbol{n}_{j(i, l)}^{\boldsymbol{o}}=\mathrm{N}_{\mathrm{j}(\mathrm{i}, \mathrm{l})} \boldsymbol{n}_{\boldsymbol{j}(\mathrm{i}, \mathrm{l})}
$$

\subsection{Discretized momentum equations}

Since momentum equations (2) and (3) are invariant under solid rotation of the $x$ and $y$ axis on the horizontal plane, a consistent semi-implicit finite volume discretization for the velocity component normal to each edge can be written as 


$$
\begin{aligned}
& U_{j, k}^{n+1}-\Delta t \operatorname{VDiff}\left(U_{j, k}^{n+1}\right)+\left.(1-\theta) \Delta \operatorname{tg} \frac{\partial \eta}{\partial n}\right|_{n}+\left.\theta \Delta \operatorname{tg} \frac{\partial \eta}{\partial n}\right|_{n+1} \\
& =U_{j, k}^{n}+\frac{\Delta t}{2}\left(3 F_{j, k}^{n}-F_{j, k}^{n-1}\right)
\end{aligned}
$$

where $\operatorname{Diff}($ ) is the centered implicated discretizaton of vertical eddy viscosity term; $\theta$ is an implicitness factor to be taken in the range $1 / 2 \leq \theta \leq 1$, the free surface term has been discretized semiimplicitly with the theta method (Casulli, 1990), the friction term has been discretized implicitly, and the explicit terms include advective terms and diffusion terms have been discretized with the second order Adams-Bashforth method, as in Fringer et al (2006) and Wang, et al(2010). The explicit terms are given by

$$
F_{j, k}=-\operatorname{adv}\left(U_{j, k}\right)+\operatorname{Hdiff}\left(U_{j, k}\right)+\operatorname{Baro}\left(\rho_{j, k}\right)+f v n_{x}-f u n_{y}
$$

where $\operatorname{adv}()$ and $\operatorname{Hdiff}()$ are the advection and diffusion operators, respectively, which are discretized by integrated method. Baro( ) is the baroclinic operator. The $x$ and $y$ direction velocities that are required for the Coriolis terms and advective terms are obtained by velocity reconstruction (Wang et al, 2010).

Taking into account the boundary conditions (12) and (14), Eq. (26) can be written in matrix form as

$$
\boldsymbol{A}_{j} \boldsymbol{U}_{j}=\boldsymbol{F}_{j}-\left.\theta \Delta \operatorname{tg} \frac{\partial \eta}{\partial \eta}\right|_{j} ^{n+1} \Delta \boldsymbol{Z}_{j}
$$

where $\boldsymbol{A}_{\boldsymbol{j}}, \boldsymbol{U}_{\boldsymbol{j}}, \boldsymbol{F}_{\boldsymbol{j}}$ and $\Delta \boldsymbol{Z}_{\boldsymbol{j}}$ are defined as Wang et al(2008).

\subsection{Discretized continue equation}

Integrating the continuity equation (1) from the bottom to the free-surface employed the surface and bottom kinematic boundary conditions (Eq.13 and Eq.15) yields the depth-averaged free-surface equation

$$
\frac{\partial \eta}{\partial t}+\frac{\partial}{\partial x} \int_{z_{b}}^{\eta} u d z+\frac{\partial}{\partial x} \int_{z_{b}}^{\eta} v d z=0
$$

Integrated the free surface equation over cell $i$, with assuming a constant free-surface elevation on each water column, a semi-implicit finite volume discretization within each cell is given by

$$
\begin{aligned}
& A_{i} \eta_{i}^{n+1}=A_{i} \eta_{i}^{n}-\theta \Delta t \sum_{l=1}^{E_{i}}\left[N_{i(l)} l_{j(i, l)} U_{j(i, l)}^{n+1} \cdot \Delta Z_{j(i, l)}\right] \\
& -(1-\theta) \Delta t \sum_{l=1}^{E_{i}}\left[N_{i(l)} l_{j(i, l)} U_{j(i, l)}^{n} \cdot \Delta Z_{j(i, l)}\right]
\end{aligned}
$$

Substitution of the expressions for $\boldsymbol{U}_{\boldsymbol{j}}^{\boldsymbol{n}+\mathbf{1}}$ from Equation (28) into (30) yields a discrete wave equation for $\eta_{i}^{n+1}$, which is given by 


$$
\begin{aligned}
& A_{i} \eta_{i}^{n+1}-(\theta \Delta t)^{2} g \sum_{l=1}^{E_{i}}\left[\left.N_{i(l)} l_{j(i, l)} \frac{\partial \eta}{\partial n}\right|_{n+1} A^{-1} \Delta \boldsymbol{Z} \cdot \Delta Z\right] \\
& =A_{i} \eta_{i}^{n}-\theta \Delta t \sum_{l=1}^{E_{i}}\left[N_{i(l)} l_{j(i, l)} A^{-1} \boldsymbol{F} \cdot \Delta Z\right]-(1-\theta) \Delta t \sum_{l=1}^{E_{i}}\left[N_{i(l)} l_{j(i, l)} U_{j(i, l)}^{n} \cdot \Delta Z_{j(i, l)}\right]
\end{aligned}
$$

\subsection{Discretized transport equation}

The general advection-diffusion equation for an arbitrary scalar quantity $\phi$, which may be either $s, k^{2}$ or $k^{2} l$, is given by

$$
\begin{aligned}
& \frac{\partial \phi}{\partial t}+\frac{\partial u \phi}{\partial x}+\frac{\partial v \phi}{\partial y}++\frac{\partial w \phi}{\partial z} \\
& =\frac{\partial}{\partial x}\left(D_{h} \frac{\partial \phi}{\partial x}\right)+\frac{\partial}{\partial y}\left(D_{h} \frac{\partial \phi}{\partial y}\right)+\frac{\partial}{\partial z}\left(D_{v} \frac{\partial \phi}{\partial z}\right)+S(\phi)
\end{aligned}
$$

where $D_{h}$ and $D_{v}$ are the horizontal and vertical eddy diffusion coefficient; $S(\phi)$ is the source term. Integrating Eq.(32) over cell $i$ with $k$ level, one can obtain the discretization equation of scalar transport, as

$$
\begin{aligned}
& -v_{i, k-1 / 2} \phi_{i, k-1}^{n+1}+\left(A_{i} \Delta z_{i, k}+v_{i, k-1 / 2}+v_{i, k+1 / 2}\right) \phi_{i}^{n+1}-v_{i, k+1 / 2} \phi_{i, k+1}^{n+1} \\
& =A_{i} \Delta z_{i, k} \phi_{i, k}^{n}+\Delta t\left[-\operatorname{adv}\left(\phi_{i, k}^{n}\right)+\operatorname{Hdiff}\left(\phi_{i, k}^{n}\right)+S\left(\phi_{i, k}^{n}\right)\right]
\end{aligned}
$$

where $v_{i, k+1 / 2}=\frac{\Delta t A_{i} D_{v i, k+1 / 2}}{\Delta z_{i, k+1 / 2}} ; \operatorname{adv}\left(\phi_{i, k}^{n}\right)$, Hdiff $\left(\phi_{i, k}^{n}\right)$ and $S\left(\phi_{i, k}^{n}\right)$ are the discretization of advection, horizontal eddy diffusion and source terms.

\section{THE ACCUMULATION PROCESS OF IMMERSED TUBE TANK OF HMZ BRIDGE}

HMZ bridge is across the Lingdingyang estuary (see Fig.1). Lingdingyang, connected with the Pearl River at its northern end and west side, is situated in the southern China (Guangdong Province). The shape of the Lingdingyan is like an inverted funnel with the narrow neck in the north and wide mouth opening to the south. Near the bridge site location, the width of Lingdingyang is about $30 \mathrm{~km}$ between Lantau Island (Hong Kong) and Macau. The north and south of Nei Lingding island is often call inner Lingdingyang and outer Lingdingyang. The water depth along the bridge varies from 0 to 30m. The immersed tube tank of HMZ bridge run across Lingding channel which is one major navigation channel for GuangZhou port, and the water depth is about $30 \mathrm{~m}$.

The tides in the Lingdingyan mainly come from the Pacific oceanic tidal propagation through the Luzon Strait (Ye and Preiffer, 1990; Mao et al, 2004) with a mean tidal range between 1.0 and $1.7 \mathrm{~m}$. They are also influenced by the geometry and bottom topography, river discharge and meteorological factors (Mao et al, 2004). The currents are rectilinear current, which is about perpendicular to the tube tank.

For simulation the accumulation process, one middle range was selected to be the three dimensional computational domain, which covers the outer Lingdingyang as depicted in Fig.3. The triangle unstructured grids are used in horizontal and the region of immersed tube tank with high resolution grids. 45 layers is divided in the vertical, the layer thickness is about $1 \mathrm{~m} \sim 2 \mathrm{~m}$. The open 
boundary condition of three-dimensional model is provided by the one and two dimensional full coupling model of Pearl River Delta (Wang et al, 2010). Two field synchronous hydrometric measurements, measured from Aug.13 to 14, 2007 and from Aug.16 to 17, 2007 in the Lingdingyang, were used for hydrodynamic verifications. For verification of the accumulation process of immersed tube tank, the numerical model was used to simulate the accumulation process of the on-site experiment tank from February 2009 to July 2009. Fig.4 shows the section bed profile compared between numerical results and field data of the on-site experiment, which shows that the numerical model can be good simulated the accumulation process. It can be seen from Fig.4 that deposited thickness of the north side of the experiment tank is bigger than south side, though the slop of the north side is less than the south side.

The immersed tube tank of HMZ bridge is about $5.76 \mathrm{~km}$ long, $47 \mathrm{~m}$ wide, $10 \mathrm{~m}$ to $43 \mathrm{~m}$ deep. Based on the verification of the $3 \mathrm{D}$ cohesive sediment model, half a year accumulation process of immersed tube tank of HMZ bridge is predicted. Fig.5 gives the cross section velocity field along the Lingdingyang navigation channel. Fig. 5 gives the average deposited thickness along the immersed tube tank, which shows that the max deposited thickness is about $1.5 \mathrm{~m}$.

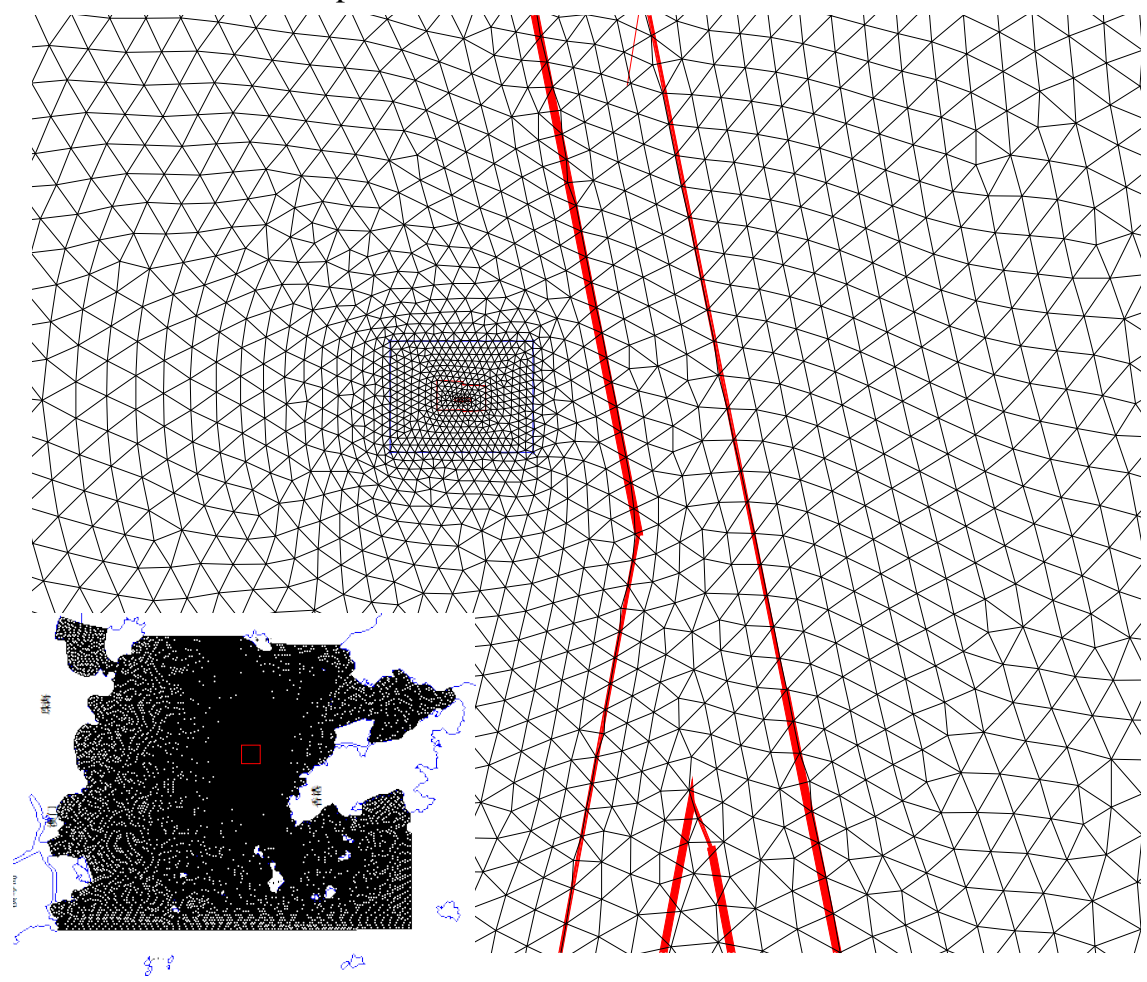

Fig.3 Sketch of 3D numerical domain and grids 


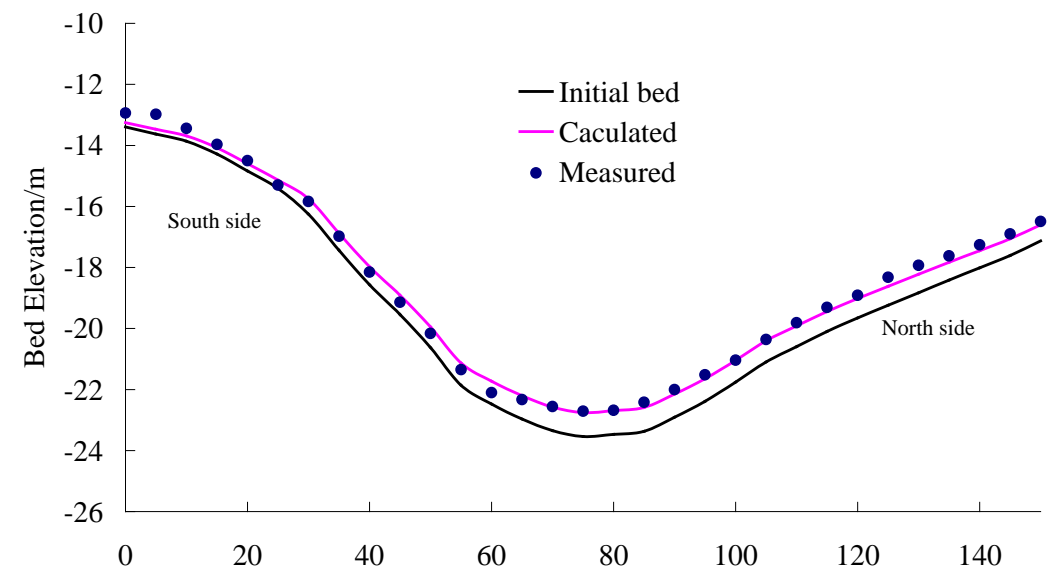

(a)

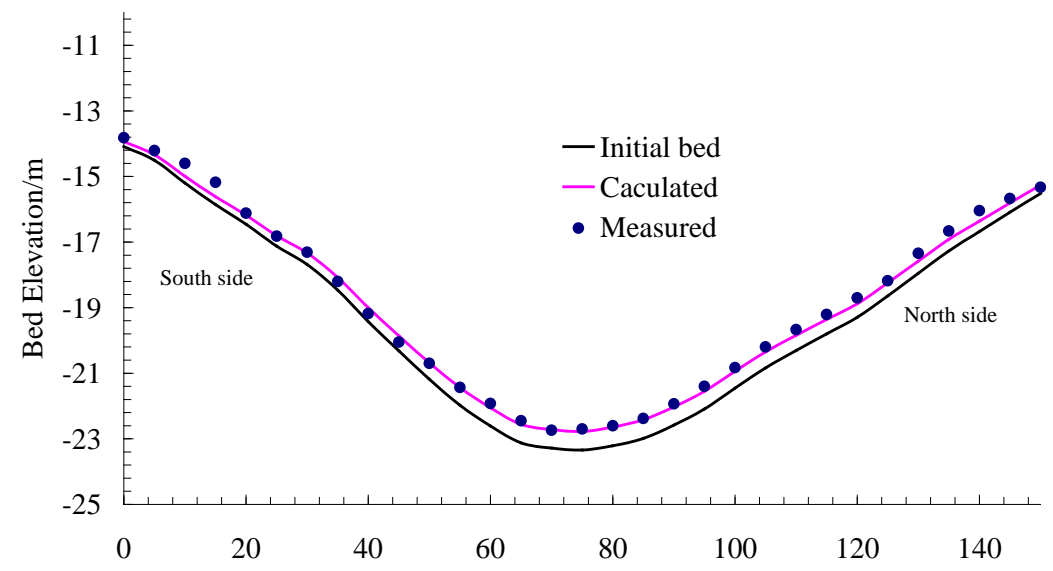

(b)

Distance/m

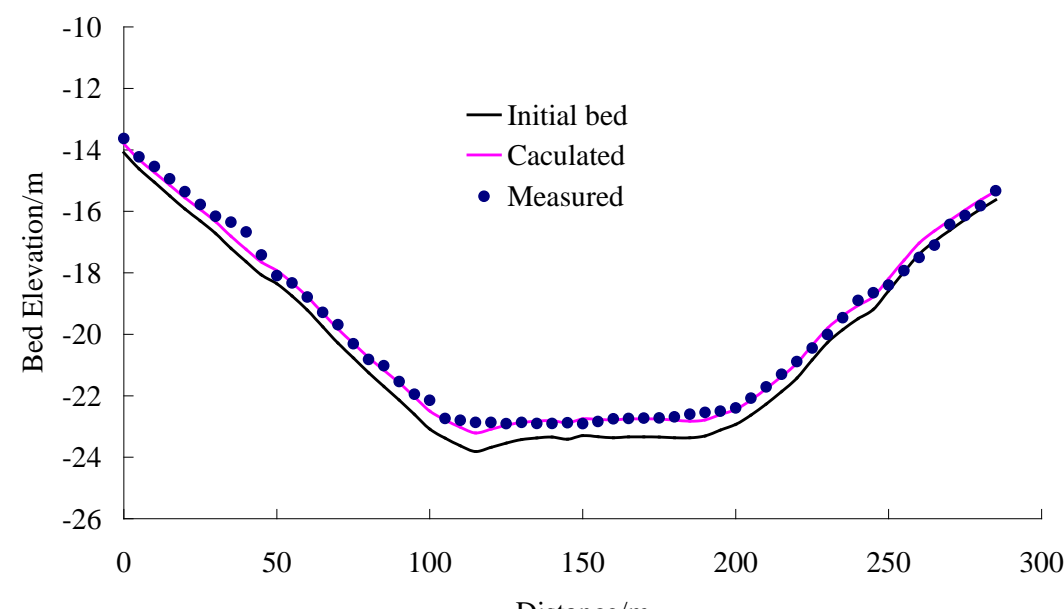

(c)

Fig.4 Bed profiles in on-Site Experiment of scoop tank: (a) SD1 section; (b) SD2 section; (C)HD1 section 


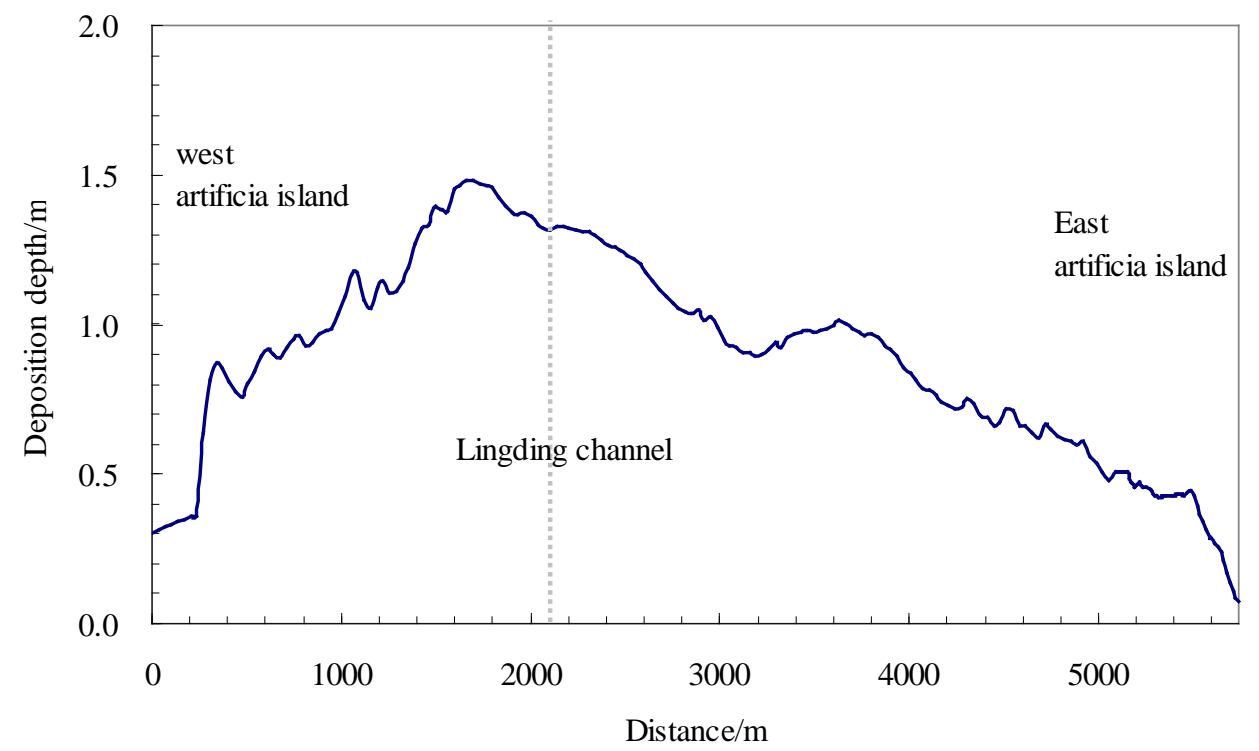

Fig.5 The deposition depth along the tunnel

\section{CONCLUSIONS}

A three dimensional unstructured cohesive sediment transport model was developed for predicting the accumulation process of immersed tube tank of HMZ bridge. Unstructured meshes provide much better representations of complex boundaries such as coastlines and islands and also provide the opportunity to concentrate mesh resolution in areas of interest. The Z-level grids and partial cell method are used in the vertical. The three dimensional model is validated by on-site experiment on immersed tube tank of HMZ bridge, the results show that the numerical can good simulate the accumulation process. Based on the validation, the model predicted the accumulation of the whole immersed tube tank of HMZ bridge.

\section{ACKNOWLEDGMENTS}

This research was supported by the National Natural Science Foundation of China under Grants 50909065 and 50879047, the technical research and development Foundation of scientific research institution under Grants 2009EG136283.

\section{REFERENCES}

Blumberg, A. F., and Mellor, G. L. 1980. A coastal ocean numerical model. Proc., Int. Symp. on Math. Modelling of Estuarine Phys., J. Sundermann and K. P. Holz, eds., Springer, Berlin, 202-19.

Blumberg, A. F., and Mellor, G. L. 1987. A description of a three dimensional coastal ocean circulation model. Three-dimensional coastal ocean models. Coastal and estuarine sciences: Volume 4, N.Heaps, ed., American Geophysical Union, Washington, D.C., 1-16.

Casulli, V., 1999. A semi-implicit finite difference method for non-hydrostatic, free-surface flows, Int. J. Numer. Methods Fluids 30, 425-440.

Chen, C.S., Liu, H.D., Beardsley, R.C., 2003. An Unstructured Grid, Finite-Volume, ThreeDimensional, Primitive Equations Ocean Model: Application to Coastal Ocean and Estuaries, Journal of Atmospheric and Oceanic Technology, 20,159-186.

Ding, Y., and Wang, S.S.Y., 2008. Recent Developments in modeling coastal and estuarine morphological processes and applications to coastal flood management and erosion protection, World Environmental and Water Resources Congress,ASCE, 1-12.

Fringer, O.B., Gerritsen, M., Street R.L., 2006. An unstructured-grid, finite-volume, nonhydrostatic, parallel coastal ocean simulator, Ocean Modelling, 14,139-173. 
Lu, Y.J., Ji, R.Y., and Zuo, L.Q., 2009. Morphodynamic responses to the deep water harbor development in the Caofeidian sea area, China's Bohai Bay, Coastal Engineering, 2009, 56(8),831-843.

Mao, Q.W., Shi,P., Yin, K.D., Gan, J.P., qi, Y.Q., 2004. Tides and tidal currents in the Pearl River Estuary. Continental Shelf Research, 24, 1797-1808.

Wang, Z.L., Lu, Y.J., and Zhou, L.Q., 2008. Unstructured 3D baroclinic model of current and salt for strong tidal estuary. The Ocean Engineering, 26(2), 43-53. (In Chinese)

Wang, Z.L. Geng, Y.F. and Lu, Y.J., 2010. 1D and 2D full coupling models for tidal flow in river networks and estuaries II: application to the Pearl River Delta. China Ocean Engineering (In press).

Ye, L., Preiffer, K.D., 0. Studies of 2D \& 3D numericalsimulation of Kelvin tide wave in Nei Lingdingyang at Pearl River Estuary. Ocean Engineering, 8 (4), 33-44. 